\title{
A.BSTRACT DIFFERENTIAL ALGEBRA AND THE ANALYTIC CASE
}

\section{A. SEIDENBERG}

In Chapter VI, Analytical considerations, of his book Differential algebra [4], J. F. Ritt states the following theorem:

Theorem. Let $Y_{i}^{p_{i}}+F_{i}, i=1, \cdots, n$, be differential polynomials in $L\left\{Y_{1}, \cdots, Y_{n}\right\}$ with each $p_{i}$ a positive integer and each $F_{i}$ either identically zero or else composed of terms each of which is of total degree greater than $p_{i}$ in the derivatives of the $Y_{j}$. Then $(0, \cdots, 0)$ is a component of the system $Y_{i}^{p_{i}}+F_{i}=0, i=1, \cdots, n$.

Here the field $L$ is not an arbitrary differential field but is an ordinary differential field appropriate to the "analytic case." ${ }^{1}$ While it lies at hand to conjecture the theorem for an arbitrary (ordinary) differential field $L$ of characteristic 0 , the type of proof given by Ritt does not place the question beyond doubt. ${ }^{2}$ The object of the present note is to establish a rather general principle, analogous to the well-known "Principle of Lefschetz" [2], whereby it will be seen that any theorem of the above type, more or less, which holds in the analytic case also holds in the abstract case. The main point of this principle is embodied in the Embedding Theorem, which tells us that any field finite over the rationals is isomorphic to a field of meromorphic functions. The principle itself can be precisely formulated as follows.

PRINCIPLE. If a theorem $T(L)$ obtains for the field $L$ provided it obtains for the subfields of $L$ finite over the rationals, and if it holds in the analytic case, then it holds for arbitrary $L$.

To illustrate, consider the theorem above. The conclusion can be formulated as follows: there exists a polynomial $G(Y) \in L\left\{Y_{1}, \cdots, Y_{n}\right\}$ with $G(0)=1$ and an integer $r$ such that $Y_{i}^{r} G(Y) \equiv 0\left[Y_{1}^{p_{1}}+F_{1}, \cdots\right.$, $\left.Y_{n}^{p_{n}}+F_{n}\right], i=1, \cdots, n$. The theorem is thus seen really to refer to the field $K$ which is obtained by adjoining to the rationals the coeffi-

Received by the editors June 10, 1957.

${ }^{1}$ Consider all the functions defined in an open region $A$, each of which can be expanded into a convergent power-series in a neighborhood of each point of $A$. Because of the connectedness of $A$, these functions form an integral domain. Any subfield of its quotient-field is a field of meromorphic functions. The "analytic case" refers to the case that the base field is a field of meromorphic functions.

2 The theorem has been established by a direct, algebraic argument in [3; p. 553]. Incidentally, we have verified that the theorem also holds in the partial differential case. 
cients of the $F_{i}$. Identifying $K$ with a field of meromorphic functions and applying the analytic case, we obtain a polynomial $G(Y)$ of the desired form, with, moreover, $G(Y) \in K\{Y\}$.

The notation is the standard notation of Ritt's book, and all page references are to that book.

Embeding Theorem. Let $R$ be the rational number-field, $K$ a finite partial differential extension-field of $R$ with $m$ types of differentiation. Then $K$ is isomorphic to a field $F$ of meromorphic functions of $m$ complex variables.

The following lemma, though nothing but a simple computational rule, will prove useful in the given formulation.

Lemma. Let $R$ be the rational number-field, $K=R\left\langle u_{1}, \cdots, u_{n}\right\rangle$ a finite ordinary differential extension-field of $R$. Let $\sigma: u_{i j} \rightarrow c_{i j}$ be an arbitrary abstract-field isomorphism of $K$ onto a field $K_{1}$. Let the field of formal power-series $K_{1}((z))$ be converted into a differential field by placing $\left(\sum a_{j} z^{j} / j !\right)^{\prime}=\sum a_{j} z^{j-1} /(j-1)$ !. Then the assignment $u_{i} \rightarrow \bar{u}_{i}$ $=\sum c_{i j} z^{i} / j$ ! defines a differential-field isomorphism of $K$ onto $R\left\langle\bar{u}_{1}, \cdots, \bar{u}_{n}\right\rangle . A$ similar statement holds for partial differential fields.

Proof. Let $H(U) \in R\left\{U_{1}, \cdots, U_{n}\right\}$, where the $U_{i}$ are indeterminates. Then $H(\bar{u})=\sum H^{(j)}(c) z^{j} / j$ !, where $H^{(j)}$ is the $j$ th derivative of $H$ and $H^{(j)}(c)=H^{(j)}(\bar{u})$ evaluated at $z=0$; or, what comes to the same thing, $H^{(j)}(c)=H_{j}\left(c_{k l}\right)$, where $H^{(j)}(U)=H_{j}\left(U_{k l}\right)$. Hence $H(\bar{u})$ $=0$ if and only if $H(u)=0$. This completes the proof in the ordinary case, and the proof for the partial case follows similarly.

Proof of THE TheOREM. The proof merely applies the lemma in such a way that the power-series converge. For the sake of exposition, we consider first the ordinary case $(m=1)$. We may assume that the differential degree of transcendency of $K / R$ is $\geqq 1$, for we may always adjoin a transcendental quantity to $K$. Using the theorem of the primitive element, ${ }^{3}$ we may therefore assume that $K$ is of the form $R\left\langle u_{1}, \cdots, u_{n}\right\rangle$, where $u_{1}, \cdots, u_{n-1}$ form a transcendency basis of $K / R$. Let $u_{n}$ satisfy an equation of order $r$, but no less, over $R\left\langle u_{1}, \cdots, u_{n-1}\right\rangle$ : say $G\left(U_{1}, \cdots, U_{n}\right)=0$ is an equation of the type specified, with $G \in R\{U\}$ and $G$ irreducible. Let complex numbers $c_{i j}$ algebraically independent over $R$ be assigned to the $U_{i j}$ occurring in $G$ with the exception of $U_{n r}$, and let $c_{n r}$ be determined from the condition $G(c)=0$. We then have $S(c) \neq 0$, where $S=\partial G / \partial U_{n r}$. Hence we

${ }^{3}$ For a simple proof of this theorem, which also holds for arbitrary $m$, see [5]; see also [1]. The use here of the primitive element is, however, merely a matter of convenience in exposition; the theorem is not used in the proof below for $m>1$. 
can solve $G(U)=0$ formally for $U_{n r}$ to obtain

$$
U_{n r}-c_{n r}=T\left(U_{i j}-c_{i j} ; U_{n 0}-c_{n 0}, \cdots, U_{n r-1}-c_{n r-1}\right)(i<n),
$$

where $T$ is a formal power-series (over the rationals) in the differences indicated. Moreover it is known that $T$ converges in some neighborhood of the specified values of the $U_{i j}$. For each $i, i=1, \cdots, n-1$, we now construct a power-series $\sum c_{i j} z^{i} / j$ !, where we use the $c_{i j}$ already considered and fill in the other $c_{i j}$ with arbitrary values subject to the following conditions: first we want all the $c_{i j}, i=1, \cdots, n-1$, $j=0,1, \cdots$ and $c_{n 0}, \cdots, c_{n r-1}$ to be algebraically independent over the rationals - this condition can certainly be met since the degree of transcendency of the complexes over the rationals is the cardinal number of the complexes; and second, we want the power-series to be convergent in some neighborhood of $z=0$-this is easily accomplished, say by making the $c_{i j}$ approach 1 . These power-series are then substituted into $T$ (with $U_{i j}$ being replaced by the $j$ th derivative of $U_{i}$ ) to obtain a function $T\left(z ; U_{n 0}-c_{n 0}, \cdots, U_{n r-1}-C_{n r-1}\right)$, which will be analytic in some neighborhood of $z=0, U_{n 0}=c_{n 0}, \cdots, U_{n r-1}=c_{n r-1}$. We now regard $U_{n r}-c_{n r}=T\left(z ; U_{n 0}-c_{n 0}, \cdots, U_{n r-1}-c_{n r-1}\right)$ as a differential equation with specified initial conditions. It is well-known that there exists a solution $\sum c_{n j} z^{j} / j$ !, where $c_{n 0}, \cdots, c_{n r}$ are as assigned. Let $v_{i}=\sum c_{i j} z^{j} / j$ !. Since $\sigma: u_{i j} \rightarrow c_{i j}$ clearly determines an abstract-field isomorphism, the assignment $u_{i} \rightarrow v_{i}$ determines, by the lemma, a differential isomorphism of $R\left\langle u_{1}, \cdots, u_{n}\right\rangle$ onto $R\left\langle v_{1}, \cdots, v_{n}\right\rangle$. This completes the proof (for $m=1$ ).

REMARK. The above argument has points of contact with the argument of Ritt on pp. 28-29.

The proof for the partial differential case proceeds on the same lines as for the ordinary case, but is more complicated due to the involved character of the necessary existence theorem for partial differential equations. Here we rely on Ritt's exposition of Riquier's existence theorem (Chapter VIII), and on the argument of pp. 172-174, which can be easily adapted to our purpose.

Let $K=R\left\langle u_{1}, \cdots, u_{n}\right\rangle$, let $u_{1}, \cdots, u_{n}$ determine the prime ideal $P$ in $R\left\{U_{1}, \cdots, U_{n}\right\}$, and let $G_{1}, \cdots, G_{s}$ be a characteristic set for $P$. Let $p_{i}$ be the leader and $S_{i}$ the separant of $G_{i}$. Consider the $U_{i j}$ (where $j$ abbreviates $j_{1} \cdots j_{m}$ ) which actually occur in the $G_{k}$. Assign complex numbers $c_{i j}$ algebraically independent over $R$ to the $U_{i j}$ with exception of the $p_{k}$. Then for these $i j, R\left(\cdots, u_{i j}, \cdots\right)$ $\cong R\left(\cdots, c_{i j}, \cdots\right)$; and this isomorphism can be extended to include all the $i j$. In this way we have complex numbers $c_{i j}$ which satisfy 
the system $G_{1}=0, \cdots, G_{s}=0, \pi S_{i} \neq 0$. Solving for the $p_{i}$ successively from $G_{1}=0, \cdots, G_{s}=0$ in the neighborhood of the $c_{i j}$, we get $p_{i}=g_{i}\left(U_{j k}-c_{j k}\right)$, where on the right occur only parametric derivatives. Then essentially by Riquier's existence theorem (see, however, also the argument of Ritt on pp. 172-174), the system $p_{i}=g_{\imath}$, considered as a system of differential equations, has a unique solution for any given initial determinations. The initial determination of $U_{i}$ is a power-series in which the terms $c_{i j_{1}} \cdots j_{m} z_{1}^{j_{1}} \cdots z_{m}^{j_{m}} / j_{1} ! \cdots j_{m}$ ! correspond to the parametric derivatives, and in which the $c_{i j}$ are arbitrary subject to the condition that the series converge in a neighborhood of $z_{1}=\cdots=z_{m}=0$. We take the $c_{i j}$ subject to this condition and in such way that they are algebraically independent over $R$. For the corresponding solution $v_{\imath}=\sum c_{i j_{1}} \cdots j_{m} z_{1}^{j_{1}} \cdots z_{m}^{j_{m}} / j_{1} ! \cdots j_{m}$ !, one finds (as for $m=1$ ) that $R\left\{u_{1}, \cdots, u_{n}\right\}$ is isomorphic to $R\left\{v_{1}, \cdots\right.$, $\left.v_{n}\right\}$. This completes the proof.

Let $K^{*}$ be a field of meromorphic functions with domain $A$, and let $B$ be an open sub-region of $A$; then the functions of $K^{*}$ restricted to $B$ form a field $K_{1}^{*}$ isomorphic to $K^{*}$. The two fields $K^{*}, K_{1}^{*}$ are frequently tacitly identified; and, in fact, this is so in the following corollary.

COROllary. Let $K, K_{1}$ be finite extensions of the rational numberfield $R, K \subseteq K_{1}$, and let $\tau: K \rightarrow K^{*}$ be an isomorphism of $K$ with a field of meromorphic functions $K^{*}$. Then $\tau$ can be extended to an isomorphism $\tau: K_{1} \rightarrow K_{1}^{*}$ of $K_{1}$ with a field of meromorphic functions $K_{1}^{*}$.

Proof. One could get a proof by modifying the above proof, letting $K$ take over the role of $R$. The result would resemble considerably Ritt's proof that a polynomial ideal $\neq(1)$, in the analytic case, has an analytic zero (see pp. 28-29, 172-174, 176). We can do perhaps a little better as follows. Let $\tau: u_{i} \rightarrow u_{i}^{*}$, where $K=R\left\langle u_{1}, \cdots, u_{n}\right\rangle$; we select some point $P$, which we may assume to be the origin, at which the $u_{i}^{*}$ are analytic (and not merely meromorphic). Continuing now first for $m=1$, the power-series $u_{i}^{*}=\sum c_{i j} z^{j} / j$ ! define an (abstract) isomorphism $\sigma: u_{i j} \rightarrow c_{i j}$ of $K$ into the complex number-field. Let $K_{1}=K\left\langle v_{1}, \cdots, v_{m}\right\rangle$ with $v_{1}, \cdots, v_{m-1}$ algebraically independent over $K$ and $v_{m}$ a primitive element of $K_{1}$ over

$$
R\left\langle u_{1}, \cdots, u_{n-1}, v_{1}, \cdots, v_{m-1}\right\rangle .
$$

Clearly $\sigma$ can be extended to $K_{1}, \sigma: v_{i j} \rightarrow d_{i j}$, with $v_{i}^{*}=\sum d_{i j} z^{j} / j$ !, $i=1, \cdots, m-1$, convergent by choice and $v_{m}^{*}=\sum d_{m j} z^{j} / j$ ! convergent by the theorem. By the lemma, $\tau^{\prime}: u_{i} \rightarrow u_{i}^{*}, v_{i} \rightarrow v_{i}^{*}$ determines an isomorphism of $K_{1}$ with $R\left\langle u_{1}^{*}, \cdots, v_{m}^{*}\right\rangle$, and clearly $\tau^{\prime}$ induces $\tau$. 
This completes the proof for $m=1$. The proof for $m>1$ is similar.

The following corollary is, as just mentioned, well-known.

Corollary. $A$ polynomial ideal $A \neq(1)$, in the analytic case, has an analytic zero.

Proof. We take a finite basis for the ideal $A$ and work over the field $K$ generated by the coefficients. There exists an abstract zero $v_{1}, \cdots, v_{m}$. By the last corollary we identify $K_{1}=K\left\langle v_{1}, \cdots, v_{m}\right\rangle$ with a field of meromorphic functions. Any sub-region $B$ (of the region for $\left.K_{1}\right)$ in which the $v_{i}$ are analytic together with the $v_{i}$ define an analytic zero of $A$.

Remarks. (1) Essentially on the basis of the above principle, the following results, established by Ritt in the analytic case, can now be considered to obtain in the abstract case (characteristic 0 ):

(a) the result on pp. 129-131,

(b) the result on pp. 144-145,

(c) the results on p. 132, $\$ 12$, provided we take as definition Ritt's characterization of a limited manifold as one which is held by a differential polynomial of the form $y^{p}+F$, where $F$ either is zero or is of degree less than $p$.

The application of the principle to these results is not absolutely automatic, but as there is no essential difficulty, we will retain our proofs in manuscript form.

(2) It would not be without significance to go over all of Ritt's results, whether established only in the analytic case or not, in order to make clear the logical character of the principle, but as the considerations can not be supported with technical difficulties, we omit them. In this connection, the reader may wish to decide whether Lefschetz's Principle applies to Lüroth's Theorem. It does apply to Hilbert's Nullstellensatz, though this point was overlooked in [6; p. 373].

(3) The above principle is not merely more difficult to establish than the corresponding principle for Algebraic Geometry, but it actually has a different character. In Algebraic Geometry, the type of theorem we have been considering can in principle be decided in a finite number of steps. That is not so in Differential Algebra: for example, given a polynomial $G$ in $R\{Y\}$, it is not known how to write down finite sets of equations for the components of $G=0$ (see pp. 177178).

\section{REFERENCES}

1. E. R. Kolchin, Extensions of differential fields, Ann. of Math. vol. 43 (1942) pp. 724-729. 
2. S. Lefschetz, Algebraic geometry, Princeton, 1953.

3. H. Levi, On the structure of differential polynomials and on their theory of ideals, Trans. Amer. Math. Soc. vol. 51 (1942) pp. 532-568.

4. J. F. Ritt, Differential algebra, Amer. Math. Soc. Colloquium Publications, vol. 33, New York, 1950.

5. A. Seidenberg, Some basic theorems in differential algebra (characteristic p, arbitrary), Trans. Amer. Math. Soc. vol. 73 (1952) pp. 174-190.

6. ——, A new decision method for elementary algebra, Ann. of Math. vol. 60 (1954) pp. 365-374.

University of California at Berkeley

\section{SOME NODAL NONCOMMUTATIVE JORDAN ALGEBRAS ${ }^{1}$}

LOUIS A. KOKORIS

1. Introduction. An algebra $A$ over a field $F$ is called a noncommutative Jordan algebra if $A$ is flexible and if the associated algebra $A^{+}$is a Jordan algebra. In a recent study by Schafer [2] of noncommutative Jordan algebras of characteristic $p$, such an algebra $A$ is called a nodal algebra if it is finite dimensional, has a unity element 1 , and has the form $A=1 F+N$ where every element of $N$ is nilpotent but $N$ is not a subalgebra of $A$. It is known [1] that nodal noncommutative Jordan algebras of characteristic zero cannot exist.

Since a noncommutative Jordan algebra is flexible, it satisfies

$$
(x, y, z)+(z, y, x)=0,
$$

where $(x, y, z)=(x y) z-x(y z)$. This is the linearized form of the flexible law $(x y) x=x(y x)$. The associated algebra $A^{+}$is obtained from the algebra $A$ by redefining multiplication by $x \cdot y=(x y+y x) / 2$. (When the characteristic of $A$ is 2 we use $x \cdot y=x y+y x$.)

In this paper we give a construction for a class of nodal noncommutative Jordan algebras for every characteristic $p \neq 2$. A subclass of these algebras consists of simple algebras and a description of ideals is given for the algebras which are not simple. For the sake of completeness, we construct a nodal algebra of characteristic 2. These examples all have the stronger property that $A^{+}$is an associative algebra.

Presented to the Society, August 30, 1957; received by the editors May 22, 1957.

1 This work was supported by the Office of Naval Research under Contract NR 043-168. 